\title{
MANEJO DEL COMPORTAMIENTO DEL. PACIENTE NIÑO EN LA CONSULTA ODONTOLÓGICA
}

\author{
'Ethman Ariel Torres M., ' $M a r t h a$ Juliana Rodríguez G., ${ }^{3}$ Olga Lucía Cañón J. \\ Grupo de Investigación: Crecimiento y desarrollo integral del niño.

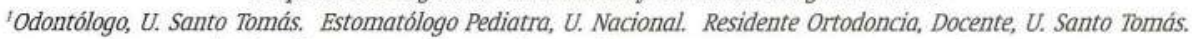 \\ ${ }^{2}$ Odontóloga, U. Javeriana Odontopediatra, C.E.S. Docente, U. SantoTomás. \\ 3odontóloga, U. Santo Tomás. Odontopediatra, U. Javeriana. Residente Ortodoncia, Docente, U. SantoTomás.
}

Autor responsable de correspondencia: Dr: Ethman Ariel Torres M.

e-mail: ethmant@ustabuca.edu.co

\begin{abstract}
RESUMEN
El manejo del comportamiento en odontología pediátrica refleja una relación continua entre el niño, sus padres y el odontólogo. Su objetivo es disminuir el miedo y la ansiedad promoviendo un proceso de entendimiento. El propósito de este documento es presentar algunas de las técnicas de manejo más usadas durante la consulta pediátrica. [Torres EA, Rodríguez MJ, Cañon OL. Manejo del comportamiento del paciente niño en la consulta odontológica. Ustasalud 2002; 1: 49-56]
\end{abstract}

PAL.ABRAS Cl.AVES: Comportamiento, Técnica, Consulta odontológica

\section{Behavior Management of the Pediatric Dental Patient}

\begin{abstract}
Behavior management is a continuum of interaction with a child, his parents and his dentist. Its goal is to reduce fear and anxiety while promoting an understanding of the need for good dental health. The purpose of this paper is to review some of the most used behavior management techniques.
\end{abstract}

KEY WORDS: Child, Behavior, Technique

Recibido para publicación: julio 17 de 2002, Aceptado para publicación: septiembre 20 de 2002

El concepto de manejo del comportamiento en odontología pediátrica se conoce a partir de los años cincuenta cuando Skinner (1) realizó un trabajo inicial al respecto. Posteriormente a éste, se ha continuado el estudio de las técnicas de manejo del comportamiento por parte de infinidad de profesionales e investigadores. ${ }^{(2-3)}$

Sawtell sugiere que la base operacional del manejo del comportamiento consiste en el uso de refuerzos seleccionados que, al ser aprendidos, transformarán el comportamiento del paciente de una forma inapropiada a una apropiada. ${ }^{(2)} \mathrm{Al}$ mencionar el término "modificación del comportamiento" es importante partir de situaciones particulares, frecuentemente presentadas durante la consulta odontológica, como sería un paciente no coopera- dor. Nathan utiliza el término precooperador y lo aplica específicamente a pacientes en edad preescolar, quienes tienen un desarrollo cognitivo inmaduro, un alto rango de restricción en sus habilidades para cooperar, mínima duración en la atención y, virtualmente, no tienen experiencia en cooperar en presencia de tensión. (3) Por estas razones, puede esperarse de ellos respuestas mal adaptativas ante situaciones que provocan ansiedad.

Los métodos de manejo del comportamiento buscan establecer la comunicación con el paciente y adicionalmente, educarlo. Por esta razón, la relación con el niño gira alrededor de un proceso dinámico de diálogo, expresión facial, tono de voz y todos aquellos métodos que emitan mensajes. ${ }^{(4)}$ Se han establecido dos objetivos principales 
en el manejo del comportamiento, que son: Realizar un tratamiento efectivo y eficiente para el niño y desarrollar en él una actitud positiva hacia el tratamiento odontológico. Dentro de los mecanismos existentes para lograrlo, se encuentran los encaminados para mantener la comunicación, mientras que otros buscan abolir los comportamientos inadecuados y enseñar al paciente cómo cooperar en el consultorio odontológico. ${ }^{(4)}$

Las técnicas empleadas tienen la gran ventaja de ser realizadas y aplicadas en forma individual a cada paciente, según sus características y requerimientos particulares. (1) No obstante existen algunas circunstancias particulares que impiden cumplir a cabalidad los objetivos de manejo del comportamiento, como alteraciones del desarrollo, retardo mental, enfermedades crónicas o agudas que impiden establecer un óptimo canal de comunicación. Esto exige buscar técnicas acordes con una cuidadosa valoración del grado de desarrollo del paciente, sus actitudes hacia el tratamiento y la predicción de la posible respuesta del niño ante la opción de tratamiento seleccionada. ${ }^{(4)}$

Todas las decisiones sobre el manejo del comportamiento se deben basar en una evaluación del balance riesgo-beneficio para el paciente, en la necesidad de tratamiento que presente, en las consecuencias de la realización del procedimiento y en el potencial de trauma físico-emocional que pueda ofrecer ${ }^{(4)}$.

\section{CONSENTIMIENTO INFORMADO}

De acuerdo con la Academia Americana de Odontología Pediátrica, es recomendable mantener informados a los padres, cuando sea necesario el uso de técnicas de manejo diferentes a aquellas en las que sólo se usa la comunicación. (4)

Carr y colaboradores encontraron que la mayoría de los odontólogos, del sureste de los Estados Unidos, buscan el consentimiento informado, verbal o escrito, cuando usan técnicas restrictivas o cuando se requiere la administración de medicamentos. ${ }^{(5)}$ El objetivo de incluir este documento dentro de la historia clínica tiene razones de tipo legal, que respaldan la obligación del profesional en relación con la información dada a los padres o tutores del paciente. ${ }^{(6,7)}$

\section{INTRODUCCIÓN A LA CONSULTA ODONTOLÓGICA}

Weinstein y Nathan ${ }^{(8)}$ consideran de vital importancia incluir dentro del proceso de iniciación del paciente pediátrico en la consulta odontológica una serie de mecanismos que incluyen:

- Orientación preventiva: Consiste en realizar citas previas al inicio del tratamiento con el fin de familiarizar al paciente con el medio ambiente odontológico, de tal forma que permita al niño enfrentar la situación que genera temor.

- Ambiente controlado y seguro: Se cumple mediante la ambientación del espacio físico que manifieste un área cálida, especialmente preparada para el niño. Para iniciar la relación odontólogo- paciente se comenzará por reconocer al niño, dirigirse a él por medio de un vocabulario acorde con su edad, transmitir importancia a su individualidad y principalmente ofrecerle confianza. ${ }^{(9)}$

- Permitir al niño la sensación de control: Lo que incluye permitir que el niño tome algunas decisiones, participar en el tratamiento y manipular algunos elementos seguros (eyector, espejo facial). De esta manera, se permite que el niño sienta que su cooperación es voluntaria y no forzada.

Es parte clave del proceso permitir al niño manifestar que siente miedo, escucharlo, hacerle saber que el operador sabe lo que está sintiendo y que se preocupa por esto. Al cuestionar su temor antes, durante y después del procedimiento se establece confianza y permite que la cooperación sea mayor que si desempeña el papel de prisionero.

Para manejar el comportamiento del paciente pediátrico, durante la consulta odontológica, se han desarrollado una variedad de técnicas, que pueden usarse de manera aislada o combinada, de acuerdo con cada individuo. El propósito de este artículo es realizar una revisión sobre las diferentes técnicas de manejo. 


\section{TÉCNICAS DE MANEjO NO FARMACOLÓGICAS}

\subsection{TÉCNICAS BÁSICAS}

\subsubsection{Comunicación verbal}

La comunicación tiene aplicación universal, es decir, puede ser utilizada con todo tipo de pacientes, cooperadores y no cooperadores, debido a que comprende la forma fundamental del manejo del comportamiento. ${ }^{(4)}$ Es necesaria para establecer relación con el niño; se considera más un proceso que una técnica, a partir de la cual surgen toda una serie de procedimientos específicos asociados para el manejo de la conducta.

\subsubsection{Control de voz}

Es una modificación controlada del tono, volumen y velocidad de la voz demostrando autoridad. ${ }^{(4,10)}$ Sus objetivos son: ganar la atención del paciente, prevenir o desviar una actitud negativa o un comportamiento evasivo y establecer autoridad. Según la Academia Americana de Odontología Pediátrica, está indicado para cualquier paciente y no tiene contraindicaciones. ${ }^{(4)}$ Sin embargo, Pinkham enfatiza en la edad del paciente, cuando se pretende utilizar esta técnica, ya que el desarrollo mental, emocional, social y cognoscitivo es el aspecto determinante del comportamiento. (11) Es importante recordar que un paciente menor de tres años no es competente en cuanto a lenguaje se refiere, razón por la cual, no será exitosamente manejado por el odontólogo que utiliza esta metodología.

\subsubsection{Comunicación no verbal}

Se refiere a la guía del comportamiento a través del contacto, la postura y la expresión facial. ${ }^{(4)}$ Pinkham la incluye como parte del manejo de la voz, y reporta que el éxito obtenido a través del control de voz no radica solamente en el volumen y en el tono convincente con que se dirija al paciente, sino también en la expresión facial que acompaña la comunicación y que va a reforzar la convicción del mensaje transmitido. ${ }^{(11)}$ Los principales objetivos de esta técnica son: Aumentar la efectividad de las demás técnicas de manejo basadas en la comunicación y ganar 0 mantener la atención del paciente. ${ }^{(4)}$ Se puede aplicar a cualquier paciente, no tiene contraindicaciones, aunque Cárdenas menciona que es especialmente útil en pacientes de temprana edad, quienes aún no han desarrollado bien la comunicación verbal. ${ }^{(12)}$

\subsubsection{Diga, Muestre, Haga ( D M H )}

Este método incluye explicaciones verbales relacionadas con los procedimientos por realizar. Primero se explica lo que se va a realizar (decir); luego se muestra (mostrar), y, por último, se realiza el procedimiento (hacer). ${ }^{(4,10,12)} \mathrm{La}$ técnica debe ser llevada a cabo de forma clara, repetitiva y cuidadosa, siendo especialmente escrupuloso en la coincidencia entre el procedimiento explicado y el ejecutado. Steelman reporta estudios en los cuales se ha demostrado que la aplicación de la técnica DMH a pacientes en edad preescolar requiere mucha más energía y talento por parte del odontólogo. ${ }^{(13)}$ Tiene la gran ventaja de transmitir al niño la sensación de confianza y tranquilidad, ya que tendrá la certeza de que no se realizarán los procedimientos hasta que él tenga conocimiento total de ellos ${ }^{(7)}$. Se recomienda su uso continuo durante toda la consulta; por lo tanto, es de gran utilidad ofrecer al paciente un espejo de cara que le permita ver el procedimiento que se está realizando. ${ }^{(12)}$ Sus objetivos son: enseñar al paciente aspectos importantes de la consulta y favorecer su familiarización con el medio. Puede ser utilizada en cualquier paciente y no tiene contraindicaciones; ${ }^{(4)}$ no obstante, Cárdenas sugiere que es poco eficaz con pacientes de muy corta edad ya que no hay un completo desarrollo de la comunicación verbal. ${ }^{(12)}$ Es una de las técnicas más utilizada por los odontólogos. ${ }^{(5,13)}$

\subsubsection{Refuerzo positivo}

Es un método efectivo para resaltar un comportamiento deseado y así fortalecer la buena conducta. ${ }^{(4)}$ Los refuerzos sociales incluyen modulación positiva de la voz, expresión facial, elogios verbales, demostraciones físicas de afecto. ${ }^{(14)}$ Los juguetes y los premios se consideran refuerzos no sociales y tienen el inconveniente que no son condicionantes, debido a que no pueden ser utilizados en forma inmediata. Su principal objetivo es reforzar un comportamiento deseado, mediante acción sobre la respuesta motora del paciente. Puede ser utilizado en todos los pacientes y no tiene contraindicaciones. ${ }^{(4,12)}$

\subsubsection{Refuerzo negativo}

Es una técnica utilizada para modificar un comportamiento no deseado mediante la expresión de rechazo. Generalmente no se utiliza aisladamente. Se usa complementándola con el control de la voz para expresar desaprobación. Idealmente debe hacerse en forma inmediata y repetida por ser un condicionamiento. No debe con- 
fundirse con una forma de castigo, algo contraindicado porque puede generar una actitud negativa ante el tratamiento y puede llegar a ser contraproducente. Cuando son utilizadas promesas para condicionar el comportamiento, como la presencia de sus padres, se debe hacer inmediatamente; debe cumplirse y no ofrecer al niño cosas imposibles. ${ }^{(14)}$ No decir la verdad o dejar de cumplir lo prometido, puede hacer que se destruya la confianza del paciente ${ }^{(12)}$.

Su objetivo principal es la modificación de un comportamiento disruptivo mediante un condicionamiento de la respuesta motora del paciente. Está indicada en pacientes que hayan desarrollado la comunicación verbal. Por lo tanto, no se debe usar con pacientes de muy corta edad, que no tengan el desarrollo adecuado de la comunicación para entender el condicionamiento. ${ }^{(12)}$

\subsubsection{Tiempo y fuera}

Es definida, por Cárdenas, como una variación del refuerzo negativo en pacientes de mayor edad. Se basa en la suspensión del tratamiento de forma temporal en la misma cita o dar por terminada la consulta pidiendo al paciente y a sus padres que regresen cuando el paciente esté preparado para cooperar. ${ }^{(12)}$ Esta técnica debe utilizarse en casos específicos en los que el paciente tenga un alto grado de desarrollo emocional y un pleno entendimiento. Además, se debe contar con la cooperación de los padres. El objetivo principal es la modificación de un comportamiento disruptivo mediante el condicionamiento y el autocontrol del paciente; de esta forma el tratamiento se condiciona a su deseo de cooperar. Está indicado en pacientes preadolescentes y adolescentes y, por lo tanto, contraindicado en pacientes que no estén en capacidad de entender la técnica por su inmadurez emocional.

\subsubsection{Distracción}

Esta técnica desvía la atención del paciente de aquello que puede ser percibido como desagradable. (4) Su objetivo es disminuir en el paciente la percepción de lo desagradable. Puede ser utilizada con cualquier paciente. Nathan la describe como técnica de distracción o diversión, siendo muy utilizada por clínicos expertos, quienes hablan al paciente en forma fluida para desviar su atención del estímulo y manipular así las percepciones y expectativas del niño. ${ }^{(3)}$
Como distractores han sido mencionadas actividades alternativas como ver televisión, videojuegos y escuchar grabaciones de cuentos o música con el fin de ganar control sobre la capacidad de respuesta del paciente. Wilson y colaboradores no encontraron disminución en la ansiedad y en el comportamiento negativo al usar la música como distractor en niños de 4 a 6 años. A pesar de esto, los pacientes solicitaron la música en sus citas posteriores. (15)

\subsubsection{Distracción contingente}

Creada por Ingersoll y colaboradores ${ }^{(16)}$ quienes desarrollaron un procedimiento distractor, que requería pocos recursos adicionales para que la conducta disruptiva del paciente cambiara, de acuerdo con algunos distractores como una cinta grabada. Es dependiente o contingente, porque se da un acceso limitado a la cinta auditiva. Al niño se le debe advertir, que puede escuchar el material grabado a través de audífonos mientras coopere con el tratamiento. Cada vez que se presente un mal comportamiento, se suspenderá la cinta la cual no comenzará nuevamente hasta observar un comportamiento adecuado. Su objetivo principal es distraer al paciente y así disminuir su ansiedad. Está indicada en cualquier paciente que haya desarrollado suficientemente su comunicación verbal. ${ }^{(12)}$

Lenis y colaboradores estudiaron 30 pacientes de comportamiento definitivamente negativo según la escala de Frankl. Se concluyó que la técnica es eficaz para ayudar a mejorar el comportamiento en algunos casos. ${ }^{(17)}$

\subsubsection{Escape contingente}

Se ha desarrollado esta técnica a partir de la premisa de que el mal comportamiento de los pacientes funciona como un escape temporal del tratamiento odontológico, y se ha reconocido que ofrecer al niño la sensación de confianza y control es una buena estrategia para lograr su cooperación. Se ofrecen breves períodos de escape del tratamiento en curso de forma contingente a partir de un comportamiento cooperador. Al permitir al niño levantar su mano recibirá un elogio y un breve escape (aproximadamente de cinco segundos) del procedimiento por permanecer tranquilo y callado. Cualquier falta de cooperación retrasará el escape hasta que se retome el comportamiento cooperador. Se ha observado en algunos estudios que el efecto preventivo de esta técnica radica en que interrumpe la cadena de ansiedad y mal compor- 
tamiento de los pacientes, mediante momentos de escape previniendo un comportamiento no cooperador. ${ }^{(18)} \mathrm{El}$ objetivo de este sistema es modificar el comportamiento disruptivo mediante el autocontrol y el condicionamiento del paciente. Está indicado en pacientes cuyo desarrollo emocional e intelectual les permita entender la técnica. Se contraindica su utilización en pacientes que no tengan la capacidad de entenderla, bien sea por su corta edad, inmadurez emocional o falta de desarrollo en la comunicación verbal. ${ }^{(19)}$

\subsubsection{Presencia / ausencia de padres}

La Academia Americana de Odontología Pediátrica la describe como una técnica que incluye el uso de la presencia o de la ausencia de los padres del niño para obtener su cooperación durante la realización del tratamiento. El objetivo de esta técnica es atraer la atención y el acatamiento por parte del paciente, prevenir o desviar el comportamiento negativo o evasivo y establecer autoridad. (4)

Continúa siendo muy polémica la decisión de permitir 0 no a los padres permanecer durante la consulta. Es importante tener en cuenta que esta decisión debe tomarse a partir del concepto del desarrollo cognoscitivo del niño según su edad, de aspectos referentes al nivel de ansiedad presente en los padres y de su posible comportamiento y del tipo de interacción entre los padres y el niño. ${ }^{(3,8)}$

Dentro de las ventajas de la permanencia de los padres en el consultorio está principalmente la contribución hecha por ellos en relación con el fortalecimiento del potencial cooperador, la oportunidad de que observen la tolerancia o la aceptación al tratamiento del paciente, ${ }^{(14)}$ aumenten la seguridad, contribuyan a familiarizar al niño con el medio ${ }^{(8)}$ y colaboren con la asistencia física, especialmente en menores de tres años. ${ }^{(3)}$ Es útil para padres escépticos que han tenido experiencias negativas en citas odontológicas anteriores. ${ }^{(20)}$

También existen razones válidas para considerar la exclusión de los padres. Se podría considerar la incapacidad de abstenerse de competir con el odontólogo en la atención del niño y la transmisión no intencionada de sus propias ansiedades por medio de palabras o lenguaje corporal. (4) En el caso de contar con la presencia de los padres, es necesario establecer las "reglas del juego", explicar claramente a los padres su papel de apoyo mientras que el niño colabore con el procedimiento, y que, en caso de ser necesario, se retiren del área de trabajo, lo que harán como parte del manejo del comportamiento de su hijo. ${ }^{(21)}$

\subsubsection{Modelado}

Es una técnica de manejo que le permite al paciente observar el tratamiento odontológico de otro paciente con buen comportamiento. ${ }^{(21)} \mathrm{Su}$ principal objetivo es disminuir la ansiedad del paciente y reforzar el comportamiento positivo. Está indicada en pacientes con un adecuado desarrollo de la comunicación verbal. ${ }^{(12)}$ Se ha observado que es especialmente útil en un rango de edad muy amplio, comprendido entre los tres y los trece años de edad. ${ }^{(18)}$

Los modelos vivos pueden llegar a ser más efectivos, si son empleados como similares, es decir, semejantes al paciente (misma edad, género, etc.). El modelado también puede realizarse con modelos simbólicos, como los títeres y las películas de video. ${ }^{(21)}$

\subsection{TÉCNICAS AVANZADAS}

La mayoría de los pacientes pueden ser manejados usando las técnicas descritas. Sólo en pocos y determinados casos se requieren técnicas más avanzadas de acuerdo con el comportamiento del paciente. Entre las técnicas avanzadas de manejo del comportamiento están: Mano sobre la boca e inmovilización. (4)

\subsubsection{Mano sobre la boca (Hom)}

Levitas, en 1.974, la denominó ejercicio de mano sobre la boca (HOME). ${ }^{(22)}$ Actualmente se define como una extensión de las formas de manejo del comportamiento por medio de la comunicación, para formular nuevamente una solicitud y volver a instaurar una apropiada comunicación. Se realiza colocando una mano sobre la boca del niño y se le explica calmadamente la expectativa de un buen comportamiento, diciéndole que la mano será retirada cuando demuestre un buen comportamiento. Al retirar la mano debe ser reforzado el buen comportamiento demostrado. (4)

Se utiliza con el fin de ganar la atención del paciente, permitir la comunicación con el odontólogo y lograr un nivel sostenido de cooperación. ${ }^{(4,7,14)}$ Adicionalmente, se busca eliminar el mal comportamiento, reemplazarlo por respuestas apropiadas aprendidas, aumentar la autoconfianza de cooperación del niño, y garantizar su seguridad durante la realización del procedimiento. ${ }^{(4)}$ Algu- 
nos autores la hacen específica para niños histéricos, agresivos, que demuestran pataletas en el sillón y son desafiantes y evasivos al tratamiento; sucede con frecuencia en niños, cuyas edades se encuentran entre tres y seis años. ${ }^{(3)}$ Debe tenerse en cuenta que es indispensable que el paciente tenga completo desarrollo de la comunicación verbal, que le permita la comprensión de la técnica. ${ }^{(12)}$

Está contraindicada en pacientes que por su edad, discapacidad, medicación o inmadurez emocional no estén en capacidad de entender y cooperar; tampoco en los casos en los que se comprometan las vías respiratorias del niño, cuando la cavidad oral sea la única vía que permita la respiración. ${ }^{(4)}$ Es necesario que al utilizar esta técnica el odontólogo no manifieste una actitud de confrontación ni enfado, ni que tenga la apariencia de aplicar una forma de castigo al paciente. (7) La aplicación de esta técnica es muy controvertida, en razón de que algunos profesionales la consideran como productora de temores, que implican posteriores evasiones ante el tratamiento. ${ }^{(3)}$ En relación con el efecto psicológico, por la aplicación de esta técnica, se ha encontrado que, aunque los pacientes recuerdan alguna experiencia médica negativa, no recuerdan el episodio como traumático. ${ }^{(23)}$ Quizás debido a esta controversia, se ha observado una disminución significativa en el número de programas de educación avanzada que enseñan esta técnica durante los últimos diez años. ${ }^{(24)}$

Es indispensable tener consentimiento informado de los padres antes de realizar la técnica. ${ }^{(4,25)}$

\subsubsection{Inmovilización fisica}

Es necesario realizar la inmovilización parcial o total de aquellos pacientes que presentan conductas combativas 0 de resistencia, para prevenir o eliminar movimientos realizados como respuestas reflejas; para proteger al paciente, al operador y al personal auxiliar de posibles injurias y para que pueda llevarse a cabo un tratamiento de calidad óptima. ${ }^{(3,4)}$ La inmovilización puede hacerse con la colaboración de los padres, del personal auxiliar o por medio de un posicionador físico. Dentro de los métodos empleados para la inmovilización se incluyen los abrebocas (de caucho y tipo trinquete) y la restricción suave y breve de la cabeza, cuerpo y extremidades en movimiento. ${ }^{(3)}$ Igualmente, se incluyen elementos de fabricación casera como sábanas con cintas de aseguramiento y aditamentos fabricados especialmente para este fin, como el Papoose Board y Pedi-Wrap, los cuales reali- zan una inmovilización más rígida y segura de cabeza y del cuerpo. ${ }^{(14,26)}$ Estos últimos son utilizados en el manejo de niños con comportamiento histérico, que interfieren la realización de los procedimientos. También se aplica para prevenir movimientos perjudiciales en pacientes con discapacidades o previamente sedados. ${ }^{(3)}$ Incluso, los pacientes con parálisis cerebral se sienten más relajados cuando sus movimientos son restringidos para la realización del procedimiento odontológico. ${ }^{(7)}$

Es de gran utilidad en procedimientos preventivos $e$ invasivos breves. ${ }^{(3,4)}$ La inmovilización está indicada para pacientes que no pueden cooperar por razones de carencia de maduración, discapacidad física o mental; en pacientes, en los que las demás opciones de manejo de comportamiento han fallado y cuando la seguridad del paciente y/o del operador podrían estar en riesgo sin el uso protector de la inmovilización. ${ }^{(4,12)}$ También, puede ser aplicado a pacientes jóvenes y a pacientes que presentan resistencia física ante el tratamiento. (7) $\mathrm{Se}$ contraindica en pacientes cooperadores y en quienes no se puede realizar una inmovilización segura, debido a condiciones médicas o sistémicas subyacentes. ${ }^{(4,12)}$ Existen reportes actuales, según los cuales los padres no aprueban la utilización de técnicas restrictivas, como el Papoose Board. Por esta razón se hace mandatoria una explicación detallada a los padres y el anexo de un documento, que incluya el consentimiento informado, el tipo de inmovilización utilizada, cómo se realiza y la duración de la aplicación. ${ }^{(4,25,26)}$

\section{TÉCNICAS DE MANEJO FARMACOLÓGICAS}

Algunas veces es necesario emplear técnicas, que usen fármacos para llevar a cabo los procedimientos odontológicos. Estas técnicas se clasifican en: Sedación consciente, sedación profunda y anestesia general. ${ }^{(27,28)}$

\subsection{SEDACIÓN CONSCIENTE}

Se define como una depresión mínima, controlada, del nivel de conciencia. El paciente mantiene sus vías aéreas intactas continuamente y puede responder a estímulos físicos y a órdenes verbales. ${ }^{27,28)}$ También se conoce como sedación interactuada. ${ }^{(29)}$

Los objetivos son reducir o eliminar la ansiedad, reducir el movimiento y posible reacción ante el tratamiento, aumentar la comunicación y cooperación del paciente, 
disminuir el umbral del dolor, incrementar la tolerancia a citas prolongadas y ofrecer la posibilidad de tratamiento de pacientes con compromiso mental, físico o médico. ${ }^{(4)}$ El profesional que utiliza esta técnica debe tener las habilidades apropiadas y las instalaciones físicas adecuadas. Además, es necesario contar con personal entrenado y equipos destinados a manejar cualquier situación de emergencia que se presente. ${ }^{(29)}$

\subsection{SEDACIÓN PROFUNDA}

Es el estado controlado de inconsciencia en el cual el paciente no se despierta fácilmente. Puede estar acompañado por pérdida parcial o total de los reflejos, incluida la inhabilidad para mantener sus vías aéreas independientes y responder a estímulos físicos o a comandos verbales. (27,28) También se conoce como sedación no interactuada, sin respuesta física. ${ }^{(29)}$

La sedación profunda se encuentra en el límite de una anestesia general, ${ }^{(27)}$ y al igual que la sedación consciente, esta técnica requiere personal altamente entrenado. $(28,29)$

\subsection{ANESTESIA GENERAL.}

Se ha definido como un estado controlado de inconsciencia acompañado por la pérdida de los reflejos protectores, incluyendo la capacidad de mantener la vía aérea independiente y responder ante la estimulación fisica y a órdenes verbales. ${ }^{(27,28)}$ Se realiza en una unidad ambulatoria o en una institución hospitalaria, ya sea con un paciente ambulatorio o con un paciente hospitalizado, según sean sus requerimientos.

Al considerar este tipo de opción de tratamiento debe tenerse en cuenta el diagnóstico del paciente, tipo de tratamiento requerido, seguridad del paciente, del operador y del equipo auxiliar, además de haber considerado todas las demás opciones de manejo del comportamiento, su salud oral, desarrollo emocional, condiciones físicas y la posibilidad de que el tratamiento bajo anestesia general pueda proteger su psiquis en desarrollo. ${ }^{(28)}$

El principal objetivo es promover la seguridad, así como también la efectividad y la eficiencia del tratamiento mediante la producción de un estado de amnesia y analgesia. Una de las indicaciones del tratamiento odontológico bajo anestesia general se da cuando el paciente necesita un tratamiento extenso y no puede cumplir de manera eficiente con un número razonable de citas utilizando o no sedación. También se usa cuando existe compromiso físico, neurológico o médico, que no permitan realizar procedimientos en el consultorio odontológico, en forma segura; en pacientes con comportamiento extremadamente ansioso, temeroso, no cooperador o fóbico, cuyo tratamiento no permita aplazamiento; en pacientes con infecciones severas, trauma facial y dental severo o limitación en el movimiento mandibular, y en pacientes para quienes la anestesia local no es efectiva debido a infección aguda, variaciones anatómicas 0 alergia. ${ }^{(28)}$

En conclusión, para el manejo del comportamiento del paciente pediátrico se debe tener en cuenta una serie de factores que hacen del tema un campo complejo. El paciente debe catalogarse de manera individual con respecto a sí mismo y a su entorno para escoger la mejor 0 las mejores técnicas de manejo que se adapten a él.

\section{BIBLIOGRAFIA}

1. Skinner BF. Science and human behavior. New York Macmillan Publ. Co. 1953.

2. Sawtell DO, Simon JF, Simonsen FJ. The effects of five preparatory methods upon child behavior during the first dental visit. J Dent Child 1974; $41: 367$ - 370 .

3. Nathan JE. Managing behavior of precooperative children. Dental Clin N Am 1995; 39: 789 - 815.

4. American Academy of Pediatric Dentistry. Guidelines for behavior management. Pediatr Dent (Spec Iss) 2001; 22: $47-51$.

5. Carr KR, Wilson S, Nimer S, Thornton JB. Behavior management techniques among pediatric dentists practicing in the southeastern United States. Pediatr Dent 1999; 21 : $347-353$.

6. Pinkham JR. Personality development and managing behavior of the cooperative preschool child. Dental Clin N America 1995; 39: 771 -787 .

7. Musselman RJ. Considerations in behavior management of the pediatric dental patient. Pediatr Clin N Am 1991; 38: 1309 - 1324.

8. Weinstein P, Nathan J. The challenge of fearful and phobic children. Dental Clin N Am 1998; 32: 667 - 692.

9. Grinberg S, Schor M. First encounter of child and dentist: An analysis of the introductory session. J Dent Child 1984; 51: 438 - 440.

10. Cárdenas D. Conducta y manejo del paciente niño. En: Fundamentos de Odontología: Odontología Pediátrica. Medellín: CIB, 1996; $22-29$. 
11. Pinkham J. Voice control. An old technique reexamined. J Dent Child 1985; 52: 199-202.

12. Cárdenas JM. Pautas para el manejo no farmacológico del comportamiento en el paciente pediátrico. Revista Academia Colombiana de Odontología Pediátrica 1997; 1: 30 - 34.

13. Steelman J. Age and sex predilection of unmanageable hispanic pediatric dental patients. J Dent Child 1991; 58: $229-232$.

14. Mathewson RJ, Primosch RE. Behavior management. En: Fundamentals of pediatric dentistry. Third Ed. Carol Stream: Quintessence Pub. 1995; 137 - 144.

15. Wilson S, Creem J, Coury D, Moursi A. The effect of music distraction on pain, anxiety and behavior in pediatric dental patients. Pediatr Dent 2002; 24: $114-118$.

16. Ingersoll BD, Nash DA, Camber C. The use of contigent audiotaped material with pediatric dental patients. J Am Dent Assoc 1984; 109: $717-719$.

17. Lenis AM, Cárdenas JM. Eficacia clínica de la técnica de distracción contingente con material auditivo en pacientes entre 4 y 5 años de edad. Revista Academia Colombiana de Odontología Pediátrica 2002; 4: $18-24$.

18. Melamed BG. A technique for reducing children in dental operatory. Dental Clin N Am 1998; 32: $693-704$.

19. Sokol DJ, Sokol S, Sokol CK. A review of nonintrusive therapies used to deal with anxiety and pain in the dental office. J Am Dent Assoc 1985; 110: $217-222$.

20. Choate BB, Seale NS, Parker WA, Wilson CF. Current trends in behavior management techniques as they relate to new standards concerning informed consent. Pediatr Dent 1990; 12: 83 - 86.

21. McTigue DJ. Behavior management of children. Dent Clin N Am 1984; 28: $81-93$.

22. Levitas TC. HOME - hand over mouth exercise. J Dent Child 1974; 41: $178-182$

23. Barton DH, Hatcher E, Potter R, Henderson HZ. Dental attitudes and memories. A study of the effects of hand over mouth/restraint. Pediatr Dent 1993; 15: 13-19.

24. Acs G, Hersch G, Testen R, Wai M. A 20 year perspective on the changing use of hand over mouth (HOM) and restraint in postdoctoral pediatric dental education. Pediatr Dent 2001; 23: 301-306.

25. Clair T. Informed consent in pediatric dentistry: A comprehensive overview. Pediatr Dent 1995: 17: 90 - 97.

26. Kuhn BR, Allen KD. Expanding child behavior management technology in pediatric dentistry: A behavioral science perspective. Pediatr Dent 1994; 16: 13 - 17.
27. Braham R, Bogetz M, Kimura M. Pharmacological patient management in pediatric dentistry. An updated. J Dent Child (Spec Iss) 1993; 60: $270-280$.

28. American Academy of Pediatric Dentistry. Guidelines for behavior management. Pediatr Dent (Spec Iss) 2001; 22: $73-78$.

29. López CZ. Guía para el manejo del paciente pediátrico bajo sedación interactuada. Revista Academia Colombiana de Odontología Pediátrica 1997; 1: 35 - 40 